with adaptation causing a rotation as well as desensitization of a set of orthogonal axes, which in their natural state are aligned along the cardinal directions. Exposing the visual system to modulation along an oblique direction might cause the original axes to realign along that direction, and those orthogonal to it, to match the new environment (see figure). At the same time, sensitivity to changes along the adapting direction are reduced: the visual system attenuates expected changes and amplifies unexpected ones. Following this argument, one might predict different results for the interaction of colour and motion when observers first adapt to fields modulated along oblique directions in colour space. Patterns formed by variations along two orthogonal cardinal directions might then cohere in motion.

Investigating the sensitivities of cell populations in the visual system should provide essential clues to the brain's colour code. Already it has been shown that in the parvocellular layers of the lateral geniculate nucleus, cell sensitivities cluster along the two cardinal directions ${ }^{\prime \prime \prime}$. In layers II and III of striate cortex, blue-yellow cells are segregated from red-green cells in metabolically active "blobs" . Other results suggest that cells in higher visual cortical areas may be broadly tuned to several directions in colour space $^{12}$. Yet the physiological data are still incomplete, and these psychophysical results emphasize that care must be taken not to perturb the colour code while deciphering it.

Anya Hurlbert is in the Department of Physiology, University of Oxford, Parks Road, Oxford OX1 3PT, UK.

\title{
Variation and vaccination
}

\author{
F.E. G. Cox
}

FOLLOWING closely on the debate ${ }^{1-t}$ about the "clonal' nature of human parasitic protozoa, the point at issue being the extent of their genetic diversity, a recent meeting* provided the chance to take stock of all aspects of variation in one of the most important groups - that whose members cause malaria.

The genetic diversity of malaria parasites, particularly of the malignant tertian form, Plasmodium falciparum, can now be analysed in great detail by molecular, biochemical and immunological techniques. From such studies, it is clear that considerable variations occur both within and between different populations, and between parasites in a single infection, and that this variation is extremely important in both drug therapy and in susceptibility to immune attack. Genetic complexity has several components: (1) allelic variation of many genes, as exemplified by the merozoite surface antigen MSA-1; (2) mutation, as exemplified by widespread resistance to drugs; (3) variation in expression of different genes, as seen in many stage-specific antigens; and (4) the occasional absence of genes due to chromosome deletion events. Added to this is recombination during meiosis in the mosquito vector, which results in the production of parasites with novel combinations of genes.

The two drugs most widely used against malaria, particularly for prophylaxis, are chloroquine and the anti-folate pyrimethamine. Resistance to them takes different forms (A. Cowman, Walter and Eliza Hall Institute of Medical Research, *Symposium on Genetic Variation of Malaria Parasites
Leiden, 14-16 November 1990. Proceedings to be published in Acta Leidensia.

Melbourne). Pyrimethamine resistance, which has multifocal origins, can be explained in terms of point mutations in the gene for dihydrofolate reductase; by contrast, resistance to chloroquine, which seems to have spread from a few foci, is thought to have a multigenic basis, although there is evidence that only a single gene is involved in a cloned line from South East Asia (T. E. Wellems, NIH, Bethesda). In Thailand (S. Thaitong, Chulalongkorn University, Bangkok) many isolates are resistant to both drugs, and clones derived from such isolates vary in their susceptibility to them. Hybridization between such drugresistant parasites in mosquitoes generates parasites with different resistance phenotypes and can give rise to multidrug-resistant forms.

\section{Diversity}

Molecular techniques can also be used to identify polymorphisms in parasite proteins, particularly the surface antigens recognized by the immune system. The extent of this diversity is astonishing - in one Sudanese village each of 29 isolates, collected within a few days of each other, contained different genotypes (D. Walliker, University of Edinburgh); and in Papua New Guinea four different kinds of the $P$. falciparum $\mathrm{S}$-antigen have been identified in a single household (R. F. Anders, Walter and Eliza Hall Institute of Medical Research, Melbourne). Similar studies have shown that in Africa and South America most infections are mixed, but in Sri Lanka most $P$. vivax infections were found to consist of single clones possibly correlated with low intensity of transmission ( $R$. Carter, University of
Edinburgh). In monkeys, changes in the antigenic profile of a cloned line of $P$. knowlesi can be caused by either a point mutation or a partial deletion of the gene for the antigen involved (Wellems). Taken together, these observations demonstrate the importance of allelic diversity, mutation and changes in gene expression in parasite variation. In the rodent malaria parasite $P$. yoelii the situation might be even more complicated, and data were presented at the meeting showing that several similar, but not identical, copies of a gene coding for a protective antigen can exist in the genome, suggesting that multigene families might be involved in the expression of variant antigens (A. A. Holder, NIMR, Mill Hill).

\section{Maintenance}

There are differing ideas as to how variant characters arise and are maintained in parasite populations - some favour random drift, others the more conventional view of selection pressure by, for example, the immune response. Given the extent of variation already recognized, there is room for both views. But one consequence is that it will be impossible to take a synoptic view of variation when designing drugs or vaccines, so for the time being it is necessary to consider a pragmatic approach. Studies on variation have also produced evidence that there are non-variable sequences for some antigens, and S. Herrera (Universidad del Valle, Colombia) discussed the possibility of using such conserved regions in "cocktail" vaccines. And in contrast to the widely held view that all the stages in the malaria life-cycle are antigenically different, certain antigens are present in more than one stage - these might be components of future vaccines (B. Sina, Biomedical Research Institute, Rockville).

The problems associated with the lack of new drugs were also covered (P. I. Trigg, WHO, Geneva; N. J. White, University of Oxford). Encouraging results have been reported for halofantrine and artemisinine derivatives, drugs that have not yet been released, but there are already reports of resistance to the most recently introduced drug, mefloquine. Given the ability of the malaria parasites to produce mutations, and to express or delete certain proteins, the development of new drugs or rational vaccines is going to be a lengthy task.

F. E. G. Cox is in the Division of Biomolecular Sciences, King's College London, Campden Hill Road, London W8 7AH, UK.

\footnotetext{
Tibayrenc, M., Kjelberg, F. \& Ayala, F. Proc. natn. Acad. Sci. U.S.A. 87, 2414-2418 (1990)

Keymer, A.E., May, R.M. \& Harvey, P.H. Nature 346, 109-110 (1990).

Walliker, D., Beale, G. \& Luzzatto, L. Nature 348, 120 (1990)

4. Dye, C.. Davies, C.R. \& Lines, J.O. Nature 348, 120 (1990)
} 